\title{
Targeted molecular profiling of genetic alterations in colorectal cancer using next-generation sequencing
}

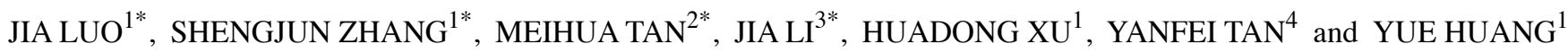 \\ ${ }^{1}$ Department of Gastroenterology, The Sanming First Hospital Affiliated to Fujian Medical University, \\ Sanming, Fujian 365000; ${ }^{2}$ BGI Education Center, University of Chinese Academy of Sciences, Beijing 100049; \\ ${ }^{3}$ Department of Thyroid and Breast, Shanghai Tenth People's Hospital, Tongji University, School of Medicine, \\ Shanghai 200072; ${ }^{4}$ Institute of Stem Cell Medicine, Fujian Medical University, Fuzhou, Fujian 350108, P.R. China
}

Received April 22, 2019; Accepted October 10, 2019

DOI: $10.3892 / 01.2019 .11203$

\begin{abstract}
Colorectal cancer (CRC) is a major contributor to cancer-associated mortality in China and remains a vast challenge worldwide. Although the genetic basis of CRC has been investigated, the uncommonly mutated genes in $\mathrm{CRC}$ remain unknown, in particular in the Asian population. In the present study, targeted region sequencing on $22 \mathrm{CRC}$ and 10 paired non-cancerous tissues was performed to determine the genetic pattern of CRC samples in the Chinese population. Driver genes were detected by three distinct softwares, including MutSigCV, oncodriveFM and iCAGES. A total of 1,335 reliable somatic mutations were identified in tumour samples compared with normal samples. Furthermore, mismatch repair (MMR) mutant patients presented significantly higher mutation density compared with MMR wild-type patients. The results from MutSigCV, oncodriveFM and iCAGES analyses simultaneously detected 29 unique driver genes. In addition, the genes $A P C$ regulator of WNT signaling pathway, $S M A D$ family member 4, neurofibromin 1, AT-rich interaction domain 5B and nuclear receptor corepressor 1 were the top five most frequently mutated genes in CRC samples, with mutation rates of 68,36 , 36,32 and $27 \%$, respectively. The findings from the present study may therefore serve as a basis for future investigation on the diagnosis and oncogenesis of CRC.
\end{abstract}

Correspondence to: Professor Yue Huang, Department of Gastroenterology, The Sanming First Hospital Affiliated to Fujian Medical University, 29 Liedong Street, Sanming, Fujian 365000, P.R. China

E-mail: smhylw@sina.com

*Contributed equally

Abbreviations: $\mathrm{CNV}$, copy number variation; CRC, colorectal cancer; GO, Gene ontology; iCAGES, Integrated CAncer GEnome Score; KEGG, Kyoto Encyclopedia of Genes and Genomes; MMR, mismatch repair

Key words: colorectal cancer, driver gene, Gene Ontology, Kyoto Encyclopedia of Genes and Genomes

\section{Introduction}

Colorectal cancer (CRC) is the fourth most frequently diagnosed cancer and the second leading cause of cancer-associated mortality worldwide according to the global cancer statistics from 2018 (1). In the United States, approximately 135,430 new cases are diagnosed each year and approximately 50,260 patients die of CRC, which accounts for approximately $9 \%$ of all cancer-associated mortalities (2). In 2015, CRC was considered as the fifth most common type of cancer in terms of occurrence and mortality in China (3). Cancer stage is a major factor contributing to the overall survival of patients with CRC. The 3-year survival rate is $80-90 \%$ for patients with CRC who are diagnosed with stages I or II, whereas the 3 -year survival rate is reduced to $20 \%$ for patients with CRC diagnosed with stage IV (4).

Over the past decade, large-scale sequencing studies have investigated the genetic basis of CRC and uncovered key pathways involved in the pathogenesis of CRC, including WNT, RAS-MAPK, PI3K, TGF- $\beta$, P53 and DNA mismatch repair pathways (5-8). The Cancer Genome Atlas program conducted a genome-scale analysis of $276 \mathrm{CRC}$ samples using multi-omics sequencing results and discovered that $16 \%$ of hypermutated CRC samples are characterized by high microsatellite instability, hypermethylation, mutL homolog $1(M L H 1)$ silencing or somatic mutations in mismatch repair genes and polymerases. In addition to the known mutations in the genes APC regulator of WNT signaling pathway $(A P C)$, tumor protein 53 (TP53), SMAD family member 4 (SMAD4), phosphatidylinositol-4,5-bisphosphate 3-kinase catalytic subunit alpha and KRAS proto-oncogene GTPase, 19 new recurrently mutated genes have been detected, including AT-rich interaction domain 1A (ARIDIA), SRY-box transcription factor 9 (SOX9) and APC membrane recruitment protein 1, and amplifications of the genes erb-b2 receptor tyrosine kinase 2 and insulin like growth factor 2 have been identified (9).

Although our understanding of genomic alterations of oncology has rapidly improved (5-9), the uncommonly mutated genes in CRC, especially in the Asian population, remain unknown. In the present study, targeted region sequencing (508 genes) on $22 \mathrm{CRC}$ and 10 paired non-cancerous samples from patients with CRC was performed to study genetic 
changes. The conclusion of this study may provide further insight into genomic changes in CRC and determine potential therapeutic targets in CRC.

\section{Materials and methods}

Patients and samples. A total of 22 primary CRC and 10 paired normal colorectal tissues were surgically resected at Sanming Hospital between May 2014 and August 2015 and immediately frozen. Patients with secondary CRC were excluded from the present study. The mean age of patients at diagnosis was $66.7 \pm 10.3$ years and ranged between 35 and 80 years old. Written informed consent was obtained from all patients and the study was approved by the Ethics Review Board of the Sanming First Hospital. The clinical characteristics of patients are presented in Table I.

DNA extraction, library preparation and sequencing. Genomic DNA was extracted from the $22 \mathrm{CRC}$ and 10 paired normal colorectal tissues using the QIAamp DNA Blood Midi Kit (Qiagen $\mathrm{GmbH}$ ) according to the manufacturer's protocol. A NanoDrop 2000 (NanoDrop Technologies; Thermo Fisher Scientific, Inc.) was used to assess DNA quality and concentration. Total DNA samples (400 ng) were used to construct sequencing libraries following the improved protocols for Illumina sequencing (10). In brief, genomic DNA samples were fragmented and ligated with Illumina standard adapters to both fragments ends. Adapter-ligated fragments are then PCR-amplified and gel-purified. Libraries were pooled (11) and hybridized using the Oseq-T panel (508 genes; BGI Genomics Co. Ltd.) for capturing. The process of hybridization was performed as previously described (12). The hybridized product was sequenced using the Illumina MiSeq platform (Illumina, Inc.) using a 100 paired-end (PE) sequencing strategy.

Variant calling and filtering. High-quality reads were aligned to the human reference genome 19 (http://hgdownload.soe. ucsc.edu/goldenPath/hg19/chromosomes/) using MEM algorithms of Burrows-Wheeler-Aligner v.0.7.17 (http://bio-bwa. sourceforge.net) with default parameters, except that the number of threads was set to 2 to accelerate this mapping process (-t 2) (13). Read deduplication was performed utilizing Picard v.1.98 software (http://picard.sourceforge.net/), using the main parameters as follows: ASSUME_SORTED=true and VALIDATION_STRINGENCY=LENIENT. Local realignment around insertion-deletions were performed using GATK v3.3.0 RealignerTargetCreator and IndelRealigner model (https://software.broadinstitute.org/gatk/) (14). Base quality score recalibration was also performed by GATK v3.3.0 BaseRecalibrator and PrintReads model (https://software.broadinstitute.org/gatk/) (13). Single nucleotide variant and insertion-deletion (indel) mutations were detected by 3.3.0 HaplotypeCaller of GATK (https://software.broadinstitute. org/gatk/). New variants and variants with maximum allele frequency $<1 \%$ reported in the 1000 genome project (15) and dbsnp v141 database (16) were considered as high confidence variants. High confidence variants that were detected specifically in CRC samples but not in normal colon tissues were regarded as somatic mutations. The SnpEff tool (http://snpeff.
sourceforge.net/SnpEff_manual.html) was applied to annotate somatic mutations (17). The final somatic variants and annotation results were used in the downstream analysis.

Driver gene prediction and functional annotation. Driver genes were predicted using three distinct computational tools, including MutSigCV (v1.0) (https://cloud.genepattern.org/gp/pages/index.jsf) (18), oncodriveFM (v0.0.1) (https://www.intogen.org) (19) and Integrated CAncer GEnome Score (iCAGES) (http://icages.wglab.org) (20). All parameters were set to default values. Driver genes were determined according to the following criteria: i) Genes with q-value $<0.1$ in the MutSigCV analysis (21); ii) genes with q-value less $<0.05$ in the oncodriveFM analysis (19); and iii) genes predicted to be drivers by iCAGES, with icagesGeneScores of predicted drivers $>0.5$ (22). To functionally annotate these driver genes, the Database for Annotation, Visualization and Integrated Discovery (https://david.ncifcrf. gov) (23) was applied to analyse the significant Gene Ontology (GO; https://david.ncifcrf.gov) terms and Kyoto Encyclopedia of Genes and Genomes (KEGG; https://david.ncifcrf.gov) (24) pathways in which driver genes were enriched. Bonferroni adjusted P-value $<0.05$ indicated a statistical significance.

Copy number variation analysis. Focal copy number variations (CNVs) were detected using CONTRA v2.0.8 software (25) between CRC and paired normal samples using default parameters, (eg: contra.py -t *.bed -s *tumor.bam -c *normal.bam -f hg19.fasta -o output-removeDups), except for '-removeDups'. For CRC samples without paired normal tissues, deduplicated bam files of 10 normal samples were merged to form one bam file. Focal CNVs were detected between deduplicated bam files of CRC samples and the merged bam file of 10 normal samples. All parameters were set to default values. Focal $\mathrm{CNVs}$ with P-values $<0.05$ were considered statistically significant. Fraction of CNV (FCNV) was computed as follows: $\mathrm{FCNV}=($ size of significant $\mathrm{CNVs}) /$ size of exons.

Statistical analyses. In the MutSigCV analysis, P-value was calculated for the gene by convoluting the background distributions of all the mutation types, and determining the probability of meeting or exceeding that score by background mutation alone. Benjamini-Hochberg false discovery rate procedure was used to correct for P-values of multiple tests. In the oncodrive FM analysis, the method randomly sampled one milliongroups of the same number of observed mutations. P-value referred to the fraction of functional impact scores equal to or greater than the observed average functional impact score of the gene. Bonferroni correction was applied to calculate the q-value for each gene. The Bonferroni correction was used to adjust the P-values computed by Fisher's exact test in the GO and KEGG pathway enrichment analyses. Difference of quantitative values was compared between two groups using Wilcoxon rank-sum test in R 3.2.0 (www.r-project.org).

\section{Results}

Clinical and demographic characteristics of the 22 patients with CRC. The 22 patients with CRC comprised 13 female and 9 male patients. The primary sites of CRC included rectum 
( 7 cases), sigmoideum ( 1 case), ascending colon ( 7 cases), transverse colon (1 case), rectosigmoid (1 case) and descending colon (5 cases). The pathological types of the 22 patients comprised 14 cases of protruding adenocarcinoma, 7 cases of protruding and mucinous adenocarcinoma and 1 case of protruding moderately differentiated adenocarcinoma. In addition, 6, 11, 3 and 2 patients with CRC were diagnosed with stages I, II, III and IV, respectively. Furthermore, 2 and 4 patients presented positive metastasis in lymph nodes and distant organs, respectively (Table I).

Summary statistics of alignment to target regions. A $3.65 \mathrm{Mb}$ target region comprising 508 genes was captured from genomic DNA and used to perform variant calling. On average, $98.87 \%$ reads were mapped successfully. The duplicated reads were removed, resulting in an average of 4,312,287 (425.73 Mb) effective reads. Of the total effective bases, $69.95 \%$ was mapped to target regions (capture specificity), and the mean sequencing depth in the target regions was 81.57 -fold. On average, $99.37 \%$ of targeted bases had at least $1 \mathrm{x}$ coverage, and $97.15 \%$ of the targeted bases had at least 10x coverage (Table SI). In addition, the distributions of per-base sequencing depth, cumulative sequencing depth and insert size are presented in Fig. 1. The density of sequencing depth on the target region followed normal distribution, with a mean of 100X. As expected, the peak of the insertion size distribution of the sequencing library was $\sim 200 \mathrm{bp}$.

Somatic mutations in colorectal cancer. To characterize the mutational spectrum, a targeted region sequencing $(3.65 \mathrm{Mb})$ on the $22 \mathrm{CRC}$ and 10 paired normal tissues was performed. A total of 1,335 somatic mutations were identified, including 122 missense mutations, 48 silent variants, 48 splicing site variants, 13 inframe deletions and 8 inframe insertions. Furthermore, 513, 404 and 113 variants were located in the untranslated region, intron and noncoding regions, respectively. In total, 52 variants caused a frame shift and 15 variants led to stop gain (Fig. 2A). The somatic mutation rates varied considerably among the samples. The average mutation density was 16.63 mutations/Mb in the 508-gene panel (range, 4.38-69.04 mutations/Mb). To analyse the cause for the different mutation densities, the mutation statuses in DNA mismatch repair (MMR) pathway genes was assessed, including $M L H 1, M L H 3$, mutS homolog 2 (MSH2), MSH3, MSH6 and PMS1 homolog 2. Five patients with CRC had mutations in any of the MMR genes, and the average mutation density in MMR mutant patients was significantly higher compared with MMR wild-type patients ( 31.73 vs. 12.18 mutations/Mb; $\mathrm{P}=0.01$; Wilcoxon rank sum test; Fig. 2B). In addition, indels, $\mathrm{C}>\mathrm{T} / \mathrm{G}>\mathrm{A}$ and $A>G / T>C$ were the three most prevalent mutation types with mutation rates of $48,18.8$, and $12.8 \%$, respectively (Fig. 2C).

Driver genes in colorectal cancer. MutSigCV analysis identified four recurrently mutated genes, named APC, TP53, SUZ12 polycomb repressive complex 2 subunit (SUZ12) and AT-rich interaction domain 5B (ARID5B), with significant statistical evidence (q-value <0.2). Furthermore, OncodriveFM and iCAGES detected five and 24 driver genes, respectively. In total, 29 unique driver genes were detected using these three computational tools. TP53 was the overlapping gene among the three sets of driver genes. APC, SMAD4, neurofibromin $1(N F 1)$,
Table I. Clinical and demographic characteristics of the 22 patients with CRC.

Variable

CRC $(n=22)$

Sex

Male 9

Female

Tumor/adenoma/Polyp location

Rectum

Sigmoideum

Ascending colon

1

Transverse colon 7

Rectosigmoid

Descending colon

1

1

5

Pathologic diagnosis

Protruding adenocarcinoma

Protruding and mucinous adenocarcinoma

Protruding moderately differentiated

7 adenocarcinoma

Pathologic stage

I

II

11

III

3

IV

Tumor stage

T1

$\mathrm{T} 2$

12

T3

2

T4

2

Nodal stage

NO

N1

1

N2

3

Metastasis stage

M0

20

M1

2

$A R I D 5 B$ and nuclear receptor corepressor 1 (NCORI) were the top five most frequently mutated genes in patients with CRC with mutation rates of $68,36,36,32$ and $27 \%$, respectively (Fig. 3). In addition, the tumour-suppressor genes APC and SMAD4 had an excessively high number of frameshift or nonsense mutations. The average mutation rate was $18 \%$ in the 29 driver genes (Table SII). Certain driver genes, including mitogen-activated protein kinase kinase kinase 1 (MAP3K1), FGFR4 and PDGFR- $\beta$, were mutated at low frequencies (5\% for all three genes), and firstly reported as driver genes in CRC samples.

To functionally annotate the 29 driver genes, GO term and KEGG pathway enrichment analyses were performed. The results from GO enrichment analysis demonstrated that the 29 driver genes were significantly enriched in 152 GO terms, including 'positive regulation of cell proliferation', 'phosphatidylinositol-mediated signalling', 'cellular response to DNA 

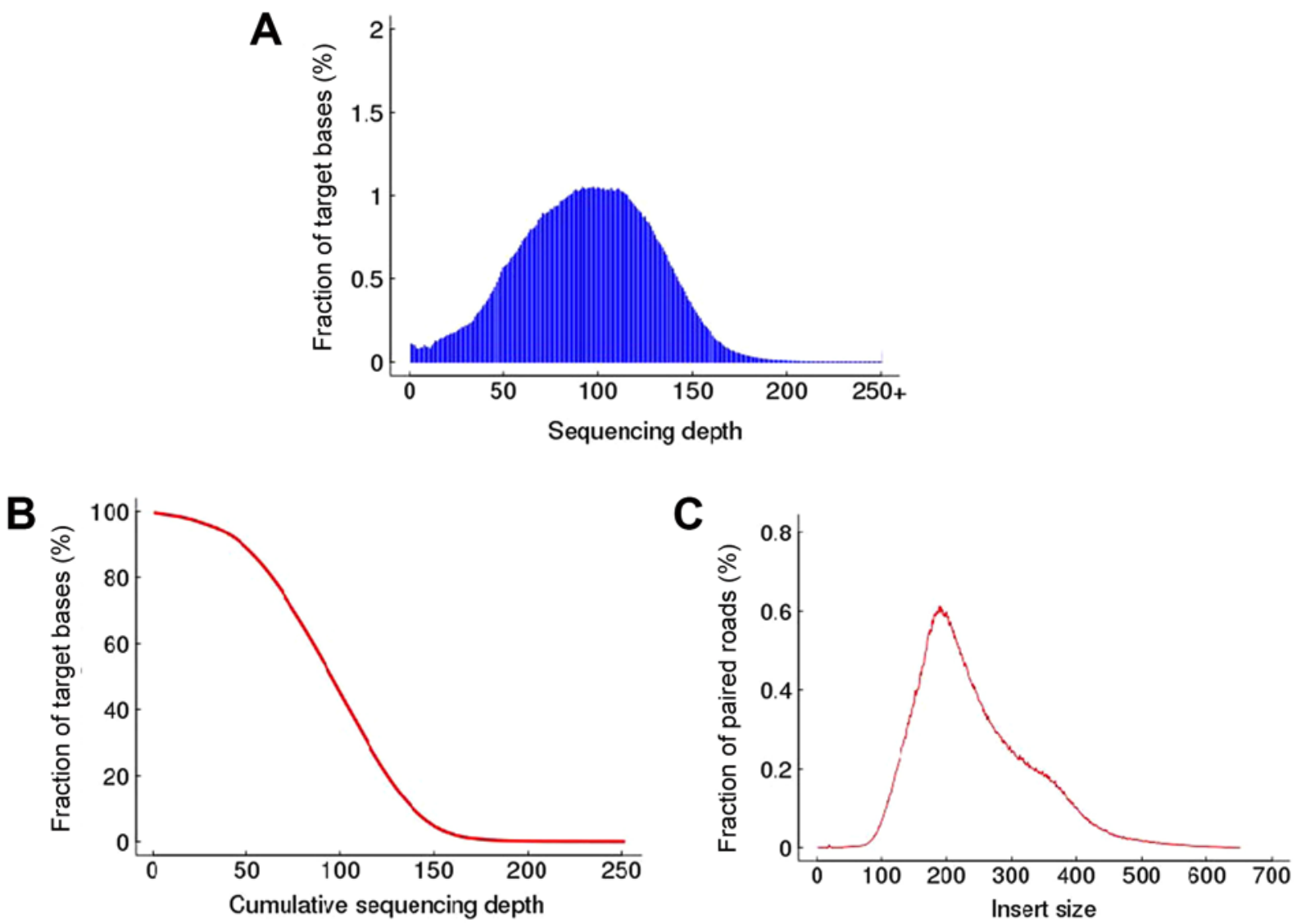

Figure 1. Distributions of per-base sequencing depth, cumulative depth and insert size of 22 colorectal cancer and 10 paired normal colon tissues. (A) Distribution of per-base sequencing depth in targeted regions. (B) Cumulative depth distribution in target regions. (C) Insert size distribution of paired reads.

damage stimulus' and 'MAPK cascade' (Bonferroni-adjusted $\mathrm{P}<0.05$; data not shown). Furthermore, the driver genes were significantly enriched in 9 KEGG pathways, including 'Wnt signalling pathways', 'cell cycle', 'colorectal cancer', 'melanoma, glioma', 'pancreatic and endometrial cancer' (Bonferroni-adjusted $\mathrm{P}<0.05$; Table SIII).

Copy number variation analysis in CRC samples. To further analyse the copy number variation in driver genes, CONTRA was used to detect focal CNVs at the exon level. The results demonstrated that 12 driver genes had FCNV $>20 \%$ in all CRC sample, including GNAS complex locus $(G N A S)$, BRCA2 DNA repair associated (BRCA2), $S O X 9$, fibroblast growth factor receptor 4 (FGFR4), SMAD4, SUZ12, FGFR3, epidermal growth factor receptor $(E G F R)$, E1A binding protein p300, NCOR1, TP53 and insulin like growth factor 1 receptor. In particular, GNAS presented a significant focal gain $>20 \%$ in $45 \%$ (10/22) of CRC samples compared with normal samples. In addition, tumour suppressor genes, including TP53, presented a significant focal loss $>20 \%$ in $22.7(5 / 22)$ and $4.5 \%(1 / 22)$ of CRC samples compared with normal samples. The EGFR oncogene exhibited a significant focal gain $>20 \%$ in $9.1 \%(2 / 22)$ of CRC samples compared with normal samples (Table II).

\section{Discussion}

The activation of driver mutations might serve a crucial role in cancer development (26). Genes that carry these driver mutations are considered as driver genes and are essential to tumorigenesis (27-31). The most common approach to predict driver genes in numerous cancer samples is the identification of genes that present significantly higher mutation frequencies compared with the background mutation rate $(18,32)$, via the MutSigCV model for example. However, many driver genes may occur at a low frequency. $(<1 \%)$ in tumours $(8)$. Novel computational tools have therefore been developed to detect driver genes with middle or low mutation frequencies. OncodriveFM first evaluates the functional impact of a somatic mutation using the three different tools SIFT (33), PolyPhen2 (34) and MutationAssessor (35), and applies the transFIC method (http://bg.upf.edu/transfic) to transform the three functional scores into a uniform score (36). To identify driver genes, oncodriveFM (19) compares the actual functional impact with a null distribution model generated by $1,000,000$ permutations and computes the bias towards the accumulation of variants with high functional impact.

The iCAGES is a novel statistical framework that can predict driver variants by integrating contributions from coding, noncoding and structural variants. iCAGES can therefore identify driver genes by combining genomic information and biological knowledge to generate prioritized drug treatments (20). iCAGES consists of three consecutive layers. The first layer prioritizes personalized cancer driver coding, noncoding and structural variations. The second layer associates these mutations to genes using a statistical model with prior biological knowledge of cancer driver genes 

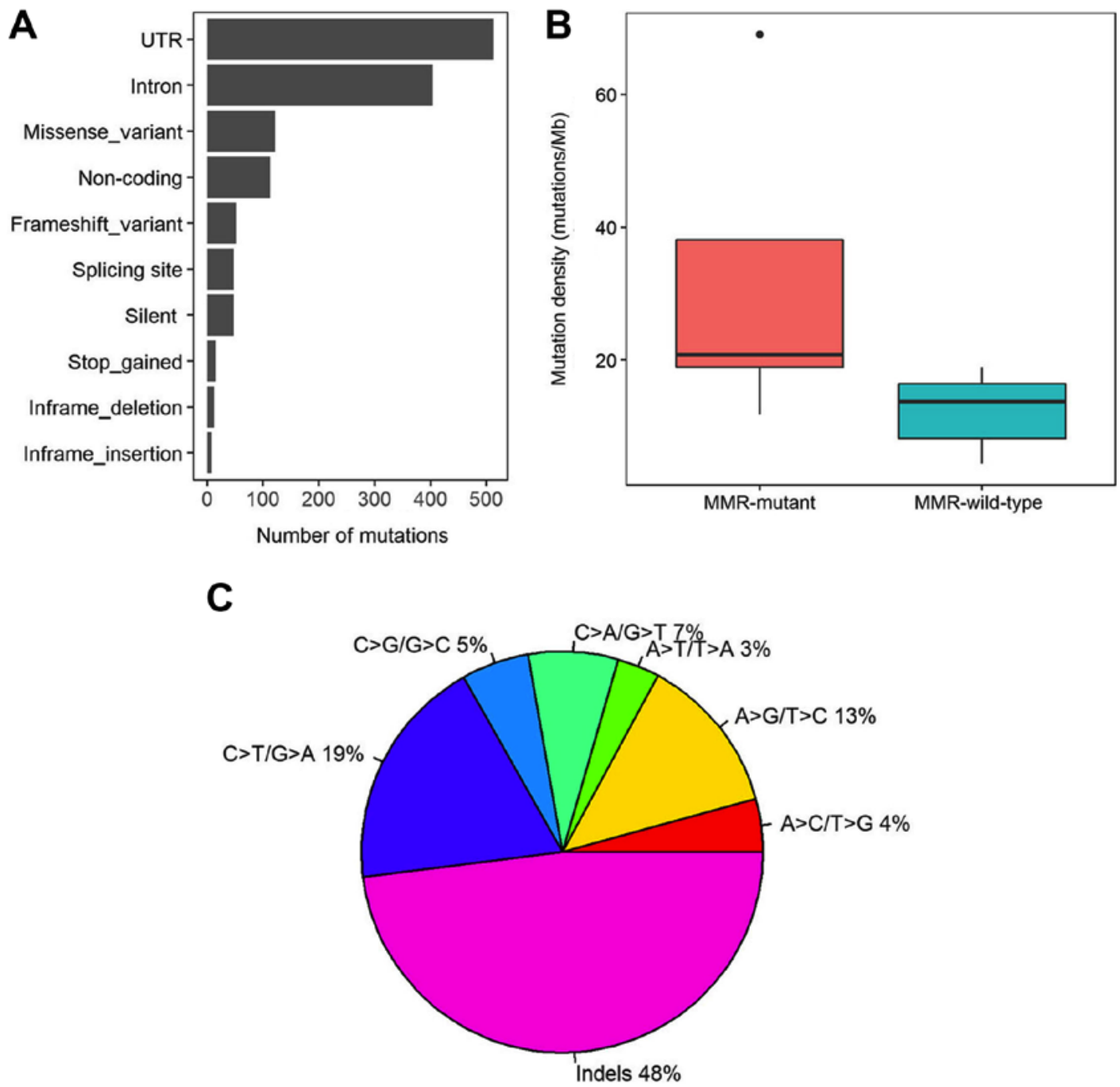

Figure 2. Characterization of somatic mutations in CRC samples. (A) Numbers of 10 different mutation classes with various functional impacts in CRC samples. (B) Differences in mutation densities between MMR mutant and MMR wild-type CRC samples. (C) Somatic mutation signatures in CRC samples. CRC, colorectal cancer; MMR, mismatch repair; UTR, untranslated region.
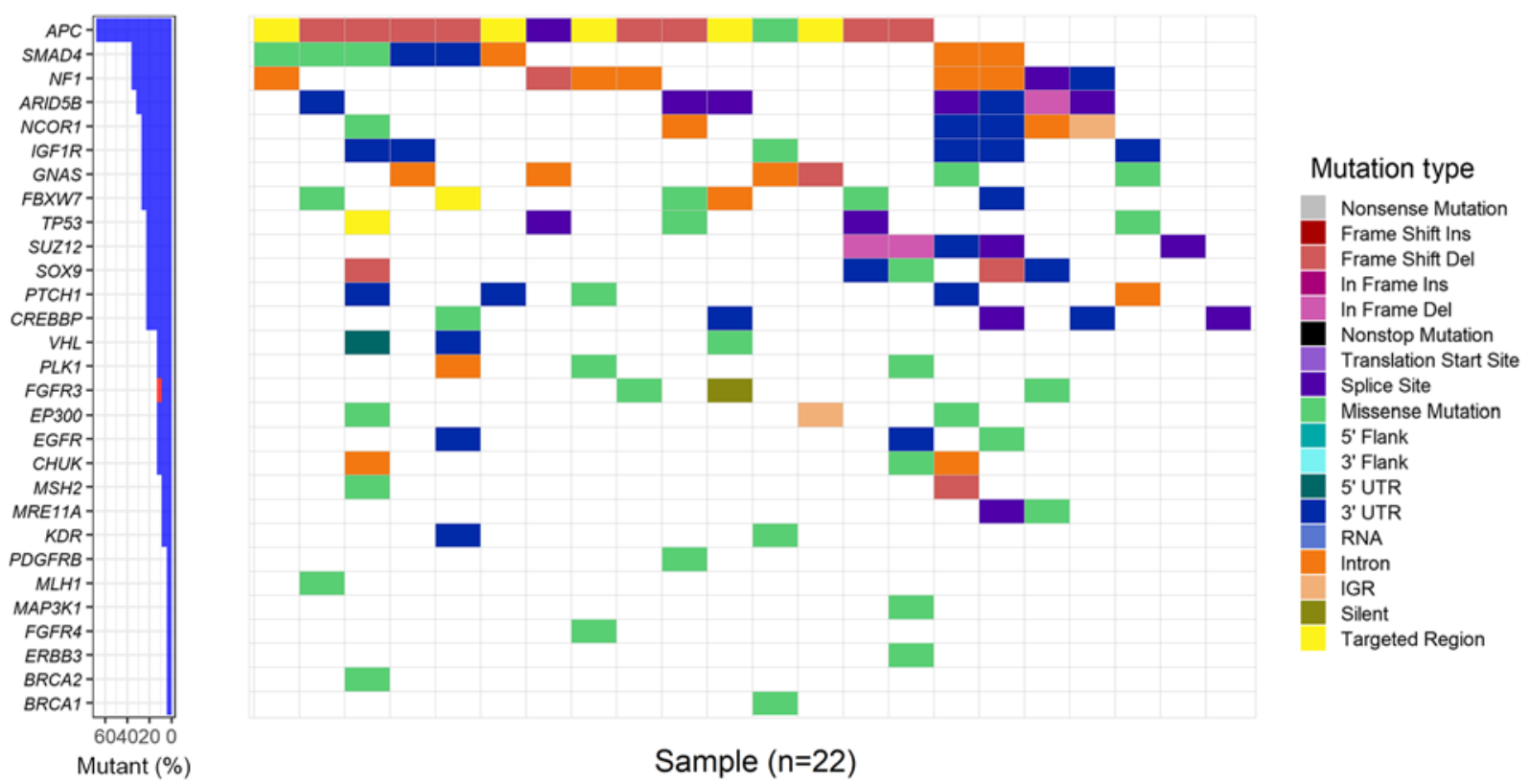

Figure 3. Mutation rates of 29 driver genes in 22 CRC samples. The left panel presents the mutation rates of 29 driver genes in CRC samples. The right panel represents the distribution of all mutations, which are coloured according to the mutation type. CRC, colorectal cancer. 
Table II. Fraction of significant CNVs of driver genes in colorectal cancer samples.

\begin{tabular}{|c|c|c|c|}
\hline Genes & Sample & Fraction of $\mathrm{CNV}$ & CNV type \\
\hline GNAS & $13 \mathrm{CB}$ & 0.54 & Gain \\
\hline GNAS & $15 \mathrm{CB}$ & 0.55 & Gain \\
\hline GNAS & $16 \mathrm{CB}$ & 0.86 & Gain \\
\hline GNAS & $18 \mathrm{CB}$ & 0.56 & Gain \\
\hline GNAS & $19 \mathrm{CB}$ & 0.56 & Gain \\
\hline GNAS & $20 \mathrm{CB}$ & 0.23 & Gain \\
\hline GNAS & $21 \mathrm{CB}$ & 0.65 & Gain \\
\hline GNAS & $31 \mathrm{CB}$ & 0.33 & Gain \\
\hline GNAS & $36 \mathrm{CB}$ & 0.56 & Gain \\
\hline GNAS & $41 \mathrm{CB}$ & 0.93 & Gain \\
\hline$B R C A 2$ & $17 \mathrm{CB}$ & 0.58 & Gain \\
\hline BRCA2 & $28 \mathrm{CB}$ & 0.88 & Gain \\
\hline$B R C A 2$ & $30 \mathrm{CB}$ & 0.56 & Gain \\
\hline$B R C A 2$ & $32 \mathrm{CB}$ & 0.49 & Loss \\
\hline BRCA2 & $35 \mathrm{CB}$ & 0.48 & Loss \\
\hline SMAD4 & $19 \mathrm{CB}$ & 0.31 & Loss \\
\hline SMAD4 & $30 \mathrm{CB}$ & 0.27 & Loss \\
\hline SMAD4 & $31 \mathrm{CB}$ & 0.22 & Loss \\
\hline SMAD4 & $40 \mathrm{CD}$ & 0.22 & Loss \\
\hline SOX9 & $17 \mathrm{CB}$ & 0.29 & Loss \\
\hline SOX9 & $18 \mathrm{CB}$ & 0.83 & Gain \\
\hline SUZ12 & $19 \mathrm{CB}$ & 0.23 & Loss \\
\hline SUZ12 & $30 \mathrm{CB}$ & 0.22 & Gain \\
\hline$F G F R 3$ & $11 \mathrm{CB}$ & 0.20 & Gain \\
\hline$F G F R 3$ & $18 \mathrm{CB}$ & 0.22 & Gain \\
\hline$E G F R$ & $16 \mathrm{CB}$ & 0.73 & Gain \\
\hline$E G F R$ & $31 \mathrm{CB}$ & 0.33 & Gain \\
\hline EP300 & $16 \mathrm{CB}$ & 0.42 & Gain \\
\hline NCORI & $19 \mathrm{CB}$ & 0.23 & Loss \\
\hline TP53 & $39 \mathrm{CB}$ & 0.27 & Loss \\
\hline$I G F I R$ & $42 \mathrm{CB}$ & 0.25 & Loss \\
\hline$F G F R 4$ & $19 \mathrm{CB}$ & 0.20 & Gain \\
\hline
\end{tabular}

$B R C A 2, \mathrm{BRCA} 2 \mathrm{DNA}$ repair associated; $\mathrm{CNV}$, copy number variation; $E G F R$, epidermal growth factor receptor; $E P 300$, E1A binding protein $\mathrm{p} 300 ; F G F R 3$, fibroblast growth factor receptor 3; FGFR4, fibroblast growth factor receptor 4; GNAS, GNAS complex locus; $I G F 1 R$, insulin like growth factor 1 receptor; NCORI, nuclear receptor corepressor 1; SMAD4, SMAD family member 4; SOX9, SRY-box transcription factor 9; $S U Z 12$, SUZ12 polycomb repressive complex 2 subunit; TP53, tumor protein $\mathrm{p} 53$.

for specific subtypes of cancer. The third layer generates a list of drugs targeting the repertoire of these potential driver genes. Three computational tools, including MutSigCV, oncodriveFM and iCAGES, were applied to detect driver genes based on complementary principles independently of somatic mutation recurrence. The combination of the three tools enables the detection of recurrently and rarely mutated driver genes in a more comprehensive manner than with MutSigCV alone (9).
Over the last decade, large-scale sequencing projects have been completed to characterize the mutation profiling of CRC $(9,37,38)$. Recurrent gene mutations, including $A P C$, KRAS proto-oncogene, GTPase and titin genes, and dysregulated signalling pathways, including the Wnt, tumour growth factor- $\beta$, phosphoinositide 3-kinase and P53 signalling pathways, have been reported to contribute to CRC carcinogenesis $(9,37,38)$. Giannakis et al (37) identified recurrently mutated genes in CRC, including BCL9 like, RNA binding motif protein 10, CCCTC-binding factor and Kruppel like factor 5, which were not previously reported in CRC. R-spondin (RSPO) gene fusions are present in $10 \%$ of colon tumours and are mutually exclusive with $A P C$ mutations, which suggests that they might be involved in the activation of Wnt signalling pathway (38). In the present study, the use of three computational algorithms, including MutSigCV, oncodriveFM and iCAGES allowed the detection of 29 driver genes on 22 CRC samples using somatic mutations. The results demonstrated that $A P C, S M A D 4, N F 1, A R I D 5 B$ and TP53 genes were frequently mutated in patients with CRC, which was similar to results from a previous study (9). Among the 29 driver genes, EGFR, kinase insert domain receptor and platelet derived growth factor receptor beta (PDGFR- $\beta$ ) are oncogenes, whereas von Hippel-Lindau tumor suppressor, NF1, SUZ12, BRCA1 and $B R C A 2$ are tumour suppressor genes, according to the curated oncogene (39) and tumour suppressor gene (40) databases. In particular, certain driver genes, including mitogen-activated protein kinase kinase kinase 1 (MAP3K1), FGFR4 and $P D G F R-\beta$, had low mutation frequencies. To the best of our knowledge, these three genes were reported as driver genes in CRC samples for the first time. MAP3K1 is a Ser/Thr protein kinase that belongs to the mitogen-activated protein kinase kinase kinase $1(\mathrm{MEKK}) /$ serine/threonine protein kinase subgroup of the MEKK family. Silencing MAP3K1 expression significantly enhances paclitaxel-induced cell proliferation inhibition in breast cancer cells, (41) pancreatic cancer cells (42) and medulloblastoma cells (43) and inhibits the human pancreatic cancer cell invasive and migratory abilities (43). In accordance to previous studies (41-44), this study suggests that $M A P 3 K 1$ may have oncogenic role in cancers. It has been demonstrated that FGFR4 serves the role of oncogene and regulator of drug or radiation resistance in CRC (45). Furthermore, FGFR4 silencing can inhibit colon cancer cell line proliferation and induces caspase-dependent apoptosis (45). In addition, FGFR4 can regulate FLICE-like inhibitory protein expression via Signal transducer and activator of transcription 3 , conferring resistance to 5 -fluorouracil (5-FU) and oxaliplatin chemotherapy in colon cancer cell lines (45). Furthermore, it has been reported that FGFR induces resistance to radiation therapy in CRC by downregulating $R A D 51$ recombinase level and inhibiting cancer cell proliferation (46). $P D G F R-\beta$ is a cell surface tyrosine kinase receptor for members of the PDGF family (47). It has been demonstrated that PDGFR- $\beta$ high expression is positively correlated with lymphatic metastasis and advanced UICC stages, and that PDGFR $-\beta$ high expression might be considered as a negative factor for the prognosis of patients with colon cancer (48-50). Decreased expression of PDGFR- $\beta$ can inhibit the invasion and proliferation of CRC cell lines (HCT116 and DLD-1) in a dose-dependent manner (48). PDGFR- $\beta$ expression may 
therefore be considered as a risk factor for recurrence in CRC, suggesting that PDGFR inhibitor could represent a useful therapeutic agent for CRC (48). These newly identified driver genes may be considered as novel candidates that could be used for functional validation in future investigation.

In conclusion, the present study screened over 500 genes in order to analyse the genetic alterations present in 22 CRC samples. A total of 29 driver genes were identified. Driver genes with significant copy number variations, such as GNAS and TP53, may be crucial in CRC oncogenesis. These findings discoveries may serve as basis for further investigation on $\mathrm{CRC}$ diagnosis and oncogenesis.

\section{Acknowledgements}

The authors would like to thank Mr. Licheng Cai and Mr. Yanyang Si from BGI-Shenzhen for their contribution on sample collection and project management.

\section{Funding}

The study was supported by the Natural Science Foundation of Fujian Province (grant nos. 2015J01568 and 2016J01656.

\section{Availability of data and materials}

The raw sequencing data that support the findings of this study have been deposited in the CNSA (https://db.cngb.org/cnsa/) of CNGBdb (accession number, CNP0000601: published on 2019/09/30).

\section{Author's contributions}

YH designed the entire study. HX, YT and SZ conducted library preparation and targeted region sequencing. JLuo, SZ, JLi and MT were responsible for the bioinformatics analyses, manuscript preparation and manuscript revision. All authors read and approved the final version of the manuscript.

\section{Ethics approval and consent to participate}

This study was approved by the Ethics Review Board of the Sanming First Hospital [SFHRB(2014) No 3] and all patients provided informed consent prior to the study.

\section{Patient consent for publication}

Not applicable.

\section{Competing interests}

The authors declare that they have no competing interests.

\section{References}

1. Bray F, Ferlay J, Soerjomataram I, Siegel RL, Torre LA and Jemal A: Global cancer statistics 2018: GLOBOCAN estimates of incidence and mortality worldwide for 36 cancers in 185 countries. CA Cancer J Clin 68: 394-424, 2018.

2. Siegel RL, Miller KD and Jemal A: Cancer statistics, 2019. CA Cancer J Clin 69: 7-34, 2019.
3. Chen W, Zheng R, Baade PD, Zhang S, Zeng H, Bray F, Jemal A, Yu XQ and He J: Cancer statistics in China, 2015. CA Cancer J Clin 66: 115-32, 2016.

4. Benitez Majano S, Di Girolamo C, Rachet B, Maringe C, Guren MG, Glimelius B, Iversen LH, Schnell EA, Lundqvist K, Christensen J, et al: Surgical treatment and survival from colorectal cancer in Denmark, England, Norway, and Sweden: A population-based study. Lancet Oncol 20: 74-87, 2019.

5. Fearon ER: Molecular genetics of colorectal cancer. Annu Rev Pathol 6: 479-507, 2011.

6. Bass AJ, Lawrence MS, Brace LE, Ramos AH, Drier Y, Cibulskis K, Sougnez C, Voet D, Saksena G, Sivachenko A, et al: Genomic sequencing of colorectal adenocarcinomas identifies a recurrent VTI1A-TCF7L2 fusion. Nat Genet 43: 964-968, 2011.

7. Sjöblom T, Jones S, Wood LD, Parsons DW, Lin J, Barber TD, Mandelker D, Leary RJ, Ptak J, Silliman N, et al: The consensus coding sequences of human breast and colorectal cancers. Science 314: 268-274, 2006.

8. Wood LD, Parsons DW, Jones S, Lin J, Sjöblom T, Leary RJ, Shen D, Boca SM, Barber T, Ptak J, et al: The genomic landscapes of human breast and colorectal cancers. Science 318: 1108-1113, 2007.

9. Cancer Genome Atlas Network: Comprehensive molecular characterization of human colon and rectal cancer. Nature 487: 330-337, 2012.

10. Bronner IF, Quail MA, Turner DJ and Swerdlow H: Improved protocols for Illumina sequencing. Curr Protoc Hum Genet 80: 18.2.1-42, 2014

11. Wei X, Sun Y, Xie J, Shi Q, Qu N, Yang G, Cai J, Yang Y, Liang Y, Wang W and Yi X: Next-generation sequencing identifies a novel compound heterozygous mutation in MYO7A in a Chinese patient with Usher Syndrome 1B. Clin Chim Acta 413: 1866-1871, 2012.

12. Shao D, Lin Y, Liu J, Wan L, Liu Z, Cheng S, Fei L, Deng R, Wang J, Chen X, et al: A targeted next-generation sequencing method for identifying clinically relevant mutation profiles in lung adenocarcinoma. Sci Rep 6: 22338, 2016.

13. Li H and Durbin R: Fast and accurate short read alignment with Burrows-Wheeler transform. Bioinformatics 25: 1754-1760, 2009.

14. McKenna A, Hanna M, Banks E, Sivachenko A, Cibulskis K, Kernytsky A, Garimella K, Altshuler D, Gabriel S, Daly M and DePristo MA: The genome analysis toolkit: A MapReduce framework for analyzing next-generation DNA sequencing data. Genome Res 20: 1297-1303, 2010.

15. Gibbs RA, Boerwinkle E, Doddapaneni H, Zhu H, Alkan C, Dal E, Kahveci F, Garrison EP, Kural D, Lee WP and Dermitzakis ET: A global reference for human genetic variation. Nature 526: 68-74, 2015

16. Sherry ST, Ward MH, Kholodov M, Baker J, Phan L, Smigielski EM and Sirotkin K: dbSNP: The NCBI database of genetic variation. Nucleic Acids Res 29: 308-311, 2001.

17. Cingolani P, Platts A, Wang le L, Coon M, Nguyen T, Wang L, Land SJ, Lu X and Ruden DM: A program for annotating and predicting the effects of single nucleotide polymorphisms, SnpEff: SNPs in the genome of Drosophila melanogaster strain w1118; iso-2; iso-3. Fly (Austin) 6: 80-92, 2012.

18. Lawrence MS, Stojanov P, Polak P, Kryukov GV, Cibulskis K, Sivachenko A, Carter SL, Stewart C, Mermel $\mathrm{CH}$, Roberts SA, et al: Mutational heterogeneity in cancer and the search for new cancer-associated genes. Nature 499: 214-218, 2013.

19. Gonzalez-Perez A and Lopez-Bigas N: Functional impact bias reveals cancer drivers. Nucleic Acids Res 40: e169, 2012.

20. Dong C, Guo Y, Yang H, He Z, Liu X and Wang K: iCAGES: Integrated CAncer GEnome Score for comprehensively prioritizing driver genes in personal cancer genomes. Genome Med 8: $135,2016$.

21. Tokheim CJ, Papadopoulos N, Kinzler KW, Vogelstein B and Karchin R: Evaluating the evaluation of cancer driver genes. Proc Natl Acad Sci USA 113: 14330-14335, 2016.

22. Zhao X, Lei Y, Li G, Cheng Y, Yang H, Xie L, Long H and Jiang R: Integrative analysis of cancer driver genes in prostate adenocarcinoma. Mol Med Rep 19: 2707-2715, 2019.

23. Huang da W, Sherman BT and Lempicki RA: Systematic and integrative analysis of large gene lists using DAVID bioinformatics resources. Nat Protoc 4: 44-57, 2009.

24. Kanehisa M, Furumichi M, Tanabe M, Sato Y and Morishima K: KEGG: New perspectives on genomes, pathways, diseases and drugs. Nucleic Acids Res 45 (D1): D353-D361, 2017. 
25. Li J, Lupat R, Amarasinghe KC, Thompson ER, Doyle MA Ryland GL, Tothill RW, Halgamuge SK, Campbell IG and Gorringe KL: CONTRA: Copy number analysis for targeted resequencing. Bioinformatics 28: 1307-1313, 2012.

26. Greenman C, Stephens P, Smith R, Dalgliesh GL, Hunter C, Bignell G, Davies H, Teague J, Butler A, Stevens C, et al: Patterns of somatic mutation in human cancer genomes. Nature 446: 153-158, 2007.

27. Barbieri CE, Baca SC, Lawrence MS, Demichelis F, Blattner M, Theurillat JP, White TA, Stojanov P, Van Allen E, Stransky N, et al: Exome sequencing identifies recurrent SPOP, FOXA1 and MED12 mutations in prostate cancer. Nat Genet 44: 685-689, 2012.

28. Grasso CS, Wu YM, Robinson DR, Cao X, Dhanasekaran SM, Khan AP, Quist MJ, Jing X, Lonigro RJ, Brenner JC, et al: The mutational landscape of lethal castration-resistant prostate cancer. Nature 487: 239-243, 2012.

29. Collisson EA, Campbell JD, Brooks AN, Berger AH, Lee W, Chmielecki J, Beer DG, Cope L, Creighton CJ, Danilova L, et al: Comprehensive molecular profiling of lung adenocarcinoma. Nature 511: 543-550, 2014.

30. Sato Y, Yoshizato T, Shiraishi Y, Maekawa S, Okuno Y, Kamura T, Shimamura T, Sato-Otsubo A, Nagae G, Suzuki H, et al: Integrated molecular analysis of clear-cell renal cell carcinoma. Nat Genet 45: 860-867, 2013.

31. Cancer Genome Atlas Research Network: Integrated genomic characterization of papillary thyroid carcinoma. Cell 159: 676-690, 2014

32. Dees ND, Zhang Q, Kandoth C, Wendl MC, Schierding W, Koboldt DC, Mooney TB, Callaway MB, Dooling D, Mardis ER, et al: MuSiC: Identifying mutational significance in cancer genomes. Genome Res 22: 1589-1598, 2012.

33. Sim NL, Kumar P, Hu J, Henikoff S, Schneider G and Ng PC: SIFT web server: Predicting effects of amino acid substitutions on proteins. Nucleic Acids Res 40 (Web Server Issue): W452-W457, 2012

34. Adzhubei IA, Schmidt S, Peshkin L, Ramensky VE, Gerasimova A, Bork P, Kondrashov AS and Sunyaev SR: A method and server for predicting damaging missense mutations. Nat Methods 7: 248-249, 2010.

35. Reva B, Antipin Y and Sander C: Predicting the functional impact of protein mutations: Application to cancer genomics. Nucleic Acids Res 39: e118, 2011.

36. González-Pérez A and López-Bigas N: Improving the assessment of the outcome of nonsynonymous SNVs with a consensus deleteriousness score, Condel. Am J Hum Genet 88: 440-449, 2011.

37. Giannakis M, Mu XJ, Shukla SA, Qian ZR, Cohen O, Nishihara R, Bahl S, Cao Y, Amin-Mansour A, Yamauchi M, et al: Genomic correlates of immune-cell infiltrates in colorectal carcinoma. Cell Rep 15: 857-865, 2016.

38. Seshagiri S, Stawiski EW, Durinck S, Modrusan Z, Storm EE, Conboy CB, Chaudhuri S, Guan Y, Janakiraman V, Jaiswal BS, et al: Recurrent R-spondin fusions in colon cancer. Nature 488: 660-664, 2012.
39. Liu Y, Sun J and Zhao M: ONGene: A literature-based database for human oncogenes. J Genet Genomics 44: 119-121, 2017.

40. Zhao M, Sun J and Zhao Z: TSGene: A web resource for tumor suppressor genes. Nucleic Acids Res 41 (Database Issue): D970-D976, 2013.

41. Hu P, Huang Q, Li Z, Wu X, Ouyang Q, Chen J and Cao Y: Silencing MAP3K1 expression through RNA interference enhances paclitaxel-induced cell cycle arrest in human breast cancer cells. Mol Biol Rep 41: 19-24, 2014.

42. Hirano T, Shino Y, Saito T, Komoda F, Okutomi Y, Takeda A, Ishihara T, Yamaguchi T, Saisho H and Shirasawa H: Dominant negative MEKK1 inhibits survival of pancreatic cancer cells. Oncogene 21: 5923-5928, 2002.

43. Antonucci L, Di Magno L, D'Amico D, Manni S, Serrao SM, Di Pastena F, Bordone R, Yurtsever ZN, Caimano M, Petroni M, et al: Mitogen-activated kinase kinase kinase 1 inhibits hedgehog signaling and medulloblastoma growth through GLI1 phosphorylation. Int J Oncol 54: 505-514, 2019.

44. Su F, Li H, Yan C, Jia B, Zhang Y and Chen X: Depleting MEKK1 expression inhibits the ability of invasion and migration of human pancreatic cancer cells. J Cancer Res Clin Oncol 135: $1655-1663,2009$

45. Turkington RC, Longley DB, Allen WL, Stevenson L, McLaughlin K, Dunne PD, Blayney JK, Salto-Tellez M, Van Schaeybroeck S and Johnston PG: Fibroblast growth factor receptor 4 (FGFR4): A targetable regulator of drug resistance in colorectal cancer. Cell Death Dis 5: e1046, 2014

46. Ahmed MA, Selzer E, Dörr W, Jomrich G, Harpain F, Silberhumer GR, Müllauer L, Holzmann K, Grasl-Kraupp B Grusch M, et al: Fibroblast growth factor receptor 4 induced resistance to radiation therapy in colorectal cancer. Oncotarget 7 : 69976-69990, 2016.

47. Heldin CH and Lennartsson J: Structural and functional properties of platelet-derived growth factor and stem cell factor receptors. Cold Spring Harb Perspect Biol 5: a009100, 2013.

48. Fujino S, Miyoshi N, Ohue M, Takahashi Y, Yasui M, Hata T, Matsuda C, Mizushima T, Doki Y and Mori M: Platelet-derived grow th factor receptor- $\beta$ gene expression relates to recurrence in colorectal cancer. Oncol Rep 39: 2178-2184, 2018.

49. Mezheyeuski A, Bradic Lindh M, Guren TK, Dragomir A, Pfeiffer P, Kure EH, Ikdahl T, Skovlund E, Corvigno S, et al: Survival-associated heterogeneity of marker-defined perivascular cells in colorectal cancer. Oncotarget 7: 41948-41958, 2016.

50. Schimanski C, Wehler T, Galle P, Gockel I and Moehler M: PDGFR- $\alpha / \beta$ expression correlates with the metastatic behavior of human colorectal cancer-A rationale for a molecular targeting strategy? J Clin Oncol 26: 22019, 2008.

This work is licensed under a Creative Commons Attribution-NonCommercial-NoDerivatives 4.0 International (CC BY-NC-ND 4.0) License. 